\title{
In Vitro Comparative Dissolution Assessment of Different Brands of Co-Amoxiclav Tablets in Pakistan
}

\author{
Muhammad Khurram Waqas ${ }^{1}$, Rehan Ali $^{1}$, Muhammad Usman ${ }^{1}$, Muhammad Nabeel \\ Shahid ${ }^{1}$, Akhtar Rasul ${ }^{2}$, Barkat Ali Khan ${ }^{3}$, and Ghulam Murtaza ${ }^{4}$ \\ ${ }^{1}$ Institute of Pharmaceutical Sciences, University of Veterinary and Animal Sciences, Lahore, Pakistan. \\ ${ }^{2}$ College of Pharmacy, Government College University, Faisalabad, Pakistan. \\ ${ }^{3}$ Faculty of Pharmacy, Gomal University, Dera Ismail Khan, Pakistan. \\ ${ }^{4}$ Department of Pharmacy, COMSATS University Islamabad, Lahore Campus, Pakistan.
}

email: gmdogar356@gmail.com

\section{ABSTRACT}

Dissolution test results are the principal indicator in estimating the in-vivo bioavailability of most oral solid dosage forms and are an important quality attribute to assess the generic formulation. This study was designed to assess the release of co-amoxiclav from finished pharmaceutical solid dosage formulations. Both innovator and generic tablets of co-amoxiclav were subjected to dissolution testing (USP method 2 and HPLC). The innovator's product (product A) met compendial criteria regarding bioavailability of both amoxicillin and clavulanic acid. The two generic brands, product $B$ and $C$, failed to meet the criteria for release of amoxicillin (i.e., $80-85 \%$ ); however, they did achieve $100 \%$ release of clavulanic acid in the initial half hour. Moreover, analysis showed significant variance between the innovator's and generic brands ( $p<0.05$ for both of amoxicillin products). Hence, the innovator's and generic brands are not bioequivalent for amoxicillin release, despite both compliance with limits for clavulanic acid release. Failure to achieve dissolution standards argues that compliance with compendial guidelines is critical for generic manufacturing of co-amoxiclav.

KEYWORDS: Generic comparison, dissolution test, bioavailability, quality assurance

\section{INTRODUCTION}

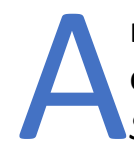

moxicillin is a broad-spectrum semisynthetic penicillin. This antibiotic exhibits good oral absorption, is structurally related to ampicillin, and effectively kills Gram-positive Streptococcal species, enterococcus species, listeria monocytogenes and Gramnegative Hemophilus influenzae, Escherichia coli, Proteus mirabilis, Salmonella species, and Shigella species (1). Clavulanic acid is a potent inhibitor of $\beta$-lactamase. It exhibits a synergistic antibiotic effect in combination with amoxicillin (2).

The synergistic effects of amoxicillin and clavulanic acid increase the susceptibility of amoxicillin towards amoxicillin-resistant $S$. aureus, enterobacteria, amoxicillin-resistant $H$. influenzae, Neisseria gonorrhoeae, and Bacteroides fragilis. The minimum inhibitory concentration (MIC) of amoxicillin usually decreases with increasing concentrations of clavulanic acid (3).

Despite good oral absorption and higher serum levels, clavulanic acid is a potent mechanism of resistance that has the potential to inactivate $\beta$-lactamase production. After identification of clavulanic acid, amoxicillin was chosen as the antibiotic, in tablet form, to be coadministered with clavulanic acid, referred to as "co-amoxiclav." This combination was introduced as Augmentin in the UK in 1981. It allowed amoxicillin/clavulanate to play an 
important role in the treatment of a wide range of infections (4). Furthermore, amoxicillin/clavulanic acid is also present in the World Health Organization (WHO) list of essential medicines (5).

In the past quarter century, dissolution testing has emerged as a highly valuable tool to characterize drug product performance. It has become an important tool in drug development and quality control. In drug development, it is used as a guide for selecting formulation parameters and for quality control of batches prior to market release. At present, dissolution testing is a requirement for market approval of almost all solid dosage formulations (6).

Drug release of an oral solid dosage form is one of the important parameters to evaluate the meaningful differences in the performance between generic and innovator brands. On that account, dissolution testing is the principal indicator in estimating in vivo bioavailability of most oral solid dosage forms (7).

The development of pharmaceutical dosage formulations also includes the evaluation of in vitro-in vivo correlation (IVIVC). Dissolution testing is also used in this context. Clinical scientists rely on dissolution testing to find the IVIVC between drug absorption in blood circulation and the release of drug from its finished solid formulation (8).

Impediments exist within dissolution testing methods. Dissolution is accepted as a representative of clinical bioavailability and the test is conducted to ensure that subsequent batches are bioequivalent (9). However, the dissolution performance itself is subject to variability both in calibration and operational characteristics of the test, thus introducing additional uncertainty.

For oral dosage forms, solubility of the drug in bodily fluid is a limiting factor for systemic absorption. Another factor is the amount of rate of drug release from a formulation (10). If two drug products with same active ingredient have similar drug concentrations at the luminal wall, they would be absorbed at the same rate and to the same extent in the blood (11). This is all the more reason to quantitate the dissolution profiles of different generic products.

In spite of the development and availability of a number of antibiotics, co-amoxiclav is still counted among the most widely used (12). In choosing an antibiotic for the treatment of an infection, efficacy is a critical parameter to be considered by prescribing physicians. In general, the innovator brand is considered to have established therapeutic efficacy, but innovator products are usually more expensive in comparison to generic brands and may not be available in developing countries. Generic drugs are thought to be biopharmaceutically similar to the corresponding innovator brand; they contain the same pharmaceutical ingredients in the same chemical form, with a similar formulation and strength and are administered via the same route (13). However, the quality of components included in the generic product may vary from the innovator brand (14).

The primary aim of this study was to compare drug release of different generic brands of coamoxiclav tablets (625 $\mathrm{mg}$ ) with innovator brand available in Pakistan.

\section{MATERIALS AND METHODS}

\section{Chemicals}

Standard drug samples of amoxicillin and clavulanate lithium were obtained from Sigma Aldrich. The generic brands chosen are the most prescribed brands in Pakistan among this 
class of antibiotics. The samples are coded as A (innovator), B, C, and D. Analytical grade monobasic sodium phosphate and methanol were acquired from Merck. Reverse osmosis grade water with conductivity less than $0.5 \mu \mathrm{S} / \mathrm{cm}$ was prepared in the pharmaceutics laboratory at University of Veterinary \& Animal Sciences, Lahore, Pakistan, using a conductivity meter (S230, Mettler Toledo).

\section{Chromatographic System}

The HPLC system (Waters) was comprised of a photodiode array (PDA) detector (operated at $220 \mathrm{~nm}$ ) and a column $4.6 \mathrm{~mm} \times 25 \mathrm{~cm} ; 10 \mu \mathrm{m}$. The mobile phase consisted of methanol and buffer (1:19), with a flow rate was set at $2 \mathrm{~mL} / \mathrm{min}$. The injection volume was $20 \mu \mathrm{L}$.

\section{HPLC Method Validation}

The USP General Information chapter <1226> provides guidelines for verifying the suitability of a compendial procedure under actual conditions of use, as stated in 21 CFR 211.194(a) (2). Method validation was done as per USP chapter $<1225>$ and $<1226>$ including various parameters as described below. Standard stock solutions of amoxicillin at $1 \mathrm{mg} / \mathrm{mL}$ and clavulanic acid at $0.4 \mathrm{mg} / \mathrm{mL}$ in water were freshly prepared. Standard solutions of amoxicillin at $0.5 \mathrm{mg} / \mathrm{mL}$ and clavulanic acid at $0.2 \mathrm{mg} / \mathrm{mL}$ in water were also freshly prepared. Sample solutions contained concentrations of amoxicillin and clavulanic acid similar to that of these standard solutions. Samples were prepared and injected into the HPLC, and results were obtained and calculated. System suitability testing was run every time to ensure the reproducibility of results.

\section{Accuracy}

Accuracy was determined by using the known purity of the reference standard and the known amount of analyte added. Accuracy was calculated as the percentage recovery from the added amount of sample using five concentration levels and duplicate injections of each in the range of $60-140 \%$ (i.e., $60 \%, 80 \%, 100 \%, 120 \%$, and $140 \%$ ) concentration of the standard for both of amoxicillin and clavulanic acid.

\section{Precision}

Precision was determined at two levels, first by calculating the intermediate precision between two analysts by comparing the relative standard deviation (RSD, \%) from six sample replicates taken from standard stock solution, and second by calculating RSD from six sample replicates. The limit for both determinations was $\pm 2 \%$ of RSD.

\section{Linearity and Range}

Linearity was established to assess the recovery for each component and within $\pm 2 \%$ of each concentration level. Linearity was established using the area of the chromatographic peaks as the function of the concentration. Various concentrations (ranging from 20\% to $200 \%$ ) of standard concentration (100\%) were analyzed using HPLC. Standard solutions were measured at various concentration levels, i.e., 20\%, 40\%,60\%, 80\%, 100\% (standard concentration), $120 \%, 140 \%, 160 \%, 180 \%$, and $200 \%$ of the target concentration. In addition, the range of concentration over which the response remained linear was also calculated. In addition, the regression equation and coefficient of correlation $\left(r^{2}\right)$ were determined by plotting the concentration ( $x$ axis) versus the mean response ( $y$ axis) for each concentration using Microsoft Excel 2010. 


\section{Dissolution Testing}

Dissolution testing was run using United States Pharmacopeia (USP) apparatus 2 (paddle method, $75 \mathrm{rpm}, 37^{\circ} \mathrm{C}$ ). A dissolution apparatus (Vision G2 Elite 8, Hanson Teledyne) with automatic tablet drop and autosampler and distilled water $(900 \mathrm{~mL})$ as dissolution medium were used in this study. The dissolution apparatus contained an automatic transfer module (syringes) with 6 dissolution vessels for instant transfer of samples for HPLC analysis. Samples were taken every 30 minutes. The not-less-than (NLT) tolerance limit for amoxicillin and clavulanic acid is $85 \%(\mathrm{Q})$ of the labeled amount to be dissolved in $30 \mathrm{~min}$.

For stage 1 (S1) testing, following the USP method for dissolution testing, each sample was tested and quantified through verified HPLC method. Six units of each sample were subjected to a single test and five batches were tested (six units from each batch). This methodology was applied to the innovator brand and each generic brand product.

\section{Disintegration Time}

Disintegration testing was performed using a basket rack assembly on a PTZ AUTO 3 (Pharma Test) per USP General Chapter of Disintegration $<701>$. Distilled water was used as a medium for disintegration maintained at $37 \pm 2{ }^{\circ} \mathrm{C}$. The instrument was programmed to run for $30 \mathrm{~min}$ as per the requirement for film-coated tablets. Disintegration time was noted when all six units completely disintegrated, leaving no residue on the sieve.

\section{Hardness Test}

Hardness test was performed using a PTB 511E (Pharma Test) per USP General Chapter on Tablet Breaking Force $<1217>$. A minimum of six tablets were used from each batch to assess the average breaking or hardness force.

\section{Statistical Analysis}

Based on these findings from five batches of each of the selected brands, the sample was subdivided into subgroups $A, B, C$, and $D$ for their percent release, and using mean value of percent release for each of individual sample. One-way analysis of variance (ANOVA) with a level of significance set at 0.05 was applied using SPSS (version 20.0) to reveal the significance of differences in mean values. Descriptive statistics were used to assess differences between and within groups for percentage of drug release, using a $95 \%$ confidence interval for amoxicillin and clavulanic acid.

\section{RESULTS AND DISCUSSION}

\section{Accuracy}

Accuracy was determined at $60 \%, 80 \%, 100 \%, 120 \%$, and $140 \%$ for amoxicillin, and the observed linearity for amoxicillin was $r^{2}=0.999$. System suitability was executed before injecting five concentration levels, and the RSD (\%) for retention time and peak area was found to be 0.137 and 0.13 , respectively. The mean tailing factor was 1.169 . These parameters meet the system suitability requirements. Recovery for amoxicillin at each level was found to be $100.35 \%, 99.45 \%, 99.65 \%, 100.04 \%$, and $100.07 \%$.

For clavulanic acid, accuracy was determined at $60 \%, 80 \%, 100 \%, 120 \%$, and $140 \%$, and the observed linearity for clavulanic acid was $r^{2}=0.999$. System suitability was run before injecting five concentration levels, and the RSD (\%) for retention time and peak area was found to be 
0.013 and 0.15 , respectively. The mean tailing factor was 1.379 . These parameters meet the system suitability requirements. Recovery for clavulanic acid at each level was calculated to be $100.24 \%, 100.17 \%, 100.10 \%, 100.47 \%$, and $100.28 \%$.

\section{Precision}

At first level, the intermediate precision was calculated between two analysts making six dilutions from the same stock solution of the same concentrations of amoxicillin. Analyst 1's results were 100.94\%, 100.83\%, 100.84\%, 100.90\%, 101.02\%, and 101.04\% (mean 100.93\%), with RSD 0.09\%. Analyst 2's results were $101.04 \%, 101.15 \%, 101.11 \%, 101.15 \%, 100.77 \%$, and $100.73 \%$ (mean $100.98 \%$ ), with RSD $0.19 \%$. These findings confirm that the parameter values lie within the acceptable limits. At the second level, the intermediate precision was also calculated. The results, calculated as a percentage of RSD, were $0.19 \%, 0.14 \%, 0.12 \%, 0.26 \%$, $0.17 \%$, and $0.55 \%$, exhibiting results within limits.

At the first level, intermediate precision was calculated between two analysts making six dilutions from the same standard stock solution of equal concentrations of clavulanic acid. Analyst 1's results were 100.04\%, 99.83\%, 99.85\%, 100.01\%, 99.84\%, and 99.94\% (mean $99.92 \%)$, with RSD $0.09 \%$. Analyst 2's results were $100.45 \%, 100.31 \%, 100.14 \%, 99.87 \%$, $99.68 \%$, and $99.93 \%$ (mean $100.06 \%$ ), with RSD $0.29 \%$, showing the parameter values to be within limits. At second level, the intermediate precision was calculated between six samples from the same vessel for each six vessels. The results calculated as RSD were $0.31 \%, 0.07 \%$, $0.43 \%, 0.07 \%, 0.42 \%$, and $0.15 \%$, exhibiting results within limits.

\section{Linearity and Range}

Linearity was studied at $20-200 \%$ of standard concentration $(100 \%)$ that was used in the sample testing by varying the injection volume of HPLC, i.e., $2-40 \mu \mathrm{L}$ of the required $20 \mu \mathrm{L}$ in quantitation of samples. The correlation coefficient $\left(r^{2}\right)$ for amoxicillin and clavulanic acid was found to be 0.9997 and 0.9998 , respectively, by plotting the percentage concentration on the $x$ axis and peak area on the $y$ axis.

\section{Limit of Detection and Limit of Quantification}

The limit of detection (LOD) and limit of quantification (LOQ) for amoxicillin were found to be 0.00250 and $0.00756 \mathrm{mg} / \mathrm{mL}$, respectively. The LOD and LOQ for clavulanic acid were found to be 0.00416 and $0.01262 \mathrm{mg} / \mathrm{mL}$, respectively.

\section{Dissolution Testing}

Results of the dissolution tests for amoxicillin are presented in Table 1, with a significant $(p<$ 0.05 ) difference noted between groups in Table 2. Results of dissolution testing and ANOVA for clavulanic acid are presented in Tables 3 and 4, respectively. Product $A$, the innovator product, met the compendial requirement, exhibiting that the release of amoxicillin and clavulanic acid was above $90 \%$ for each of the five batches. Product $B$, one of the generic local brands, exhibited a release of clavulanic acid above $100 \%$ for each of the five batches, but mean amoxicillin release for each of the five batches fell between $80-85 \%$, short of the tolerance limit of $90 \%$. This represents a compromised product formulation design. Similarly, product $C$, another local generic brand, exhibited a release of clavulanic acid above $100 \%$ for each of the five batches, but mean amoxicillin release for each of the five batches fell between $80-85 \%$, representing again a compromised product formulation design. Conversely, product $\mathrm{D}$, a generic brand from a multinational company, exhibited release of both amoxicillin and 
clavulanic acid that were above $90 \%$ for each of the five batches, showing acceptable finished product parameters.

Table 1. Descriptive Statistics for Release of Amoxicillin from Co-Amoxiclav Tablets (625 mg)

\begin{tabular}{|c|c|c|c|c|c|c|}
\hline & N & Mean (\%) & SD & SE & 95\% Cl & Range \\
\hline Product A & 5 & 94.826 & 2.534 & 1.133 & $91.680-97.972$ & $90.7-96.7$ \\
\hline Product B* & 5 & 81.640 & 2.591 & 1.159 & $78.423-84.857$ & $79.4-85.9$ \\
\hline Product C* & 5 & 83.860 & 2.344 & 1.048 & $80.950-86.770$ & $80.9-86.5$ \\
\hline Product D & 5 & 96.240 & 1.369 & 0.612 & $94.541-97.939$ & $94.0-97.3$ \\
\hline Overall & $\mathbf{2 0}$ & $\mathbf{8 9 . 1 4 2}$ & $\mathbf{6 . 9 4 5}$ & $\mathbf{1 . 5 5 3}$ & $\mathbf{8 5 . 8 9 1 - 9 2 . 3 9 2}$ & $\mathbf{7 9 . 4 - 9 7 . 3}$ \\
\hline
\end{tabular}

Note: *indicates the products which did not meet the compendial criteria. SD: standard deviation; SE: standard error, Cl: confidence interval.

Table 2. Analysis of Variance for Percent Release of Amoxicillin from Co-Amoxiclav Tablets (625 mg)

\begin{tabular}{|l|c|c|c|c|c|}
\hline & Sum of Squares & df & Mean Square & F & p. \\
\hline Between Groups & 834.345 & 3 & 278.115 & 54.267 & 0.0001 \\
\hline Within Groups & 82.000 & 16 & 5.125 & & \\
\hline Total & 916.344 & 19 & & & \\
\hline
\end{tabular}

$d f$ : degree of freedom; $F$ : $F$ is a ratio of two mean square values.

Table 3. Descriptive Statistics for Release of Clavulanic Acid from Co-Amoxiclav Tablets (625 mg)

\begin{tabular}{|c|c|c|c|c|c|c|}
\hline & N & Mean (\%) & SD & SE & $95 \%$ Cl & Range \\
\hline Product A & 5 & 97.560 & 0.737 & 0.329 & $96.645-98.475$ & $97.0-98.7$ \\
\hline Product B & 5 & 105.580 & 2.484 & 1.111 & $102.495-108.665$ & $103.7-109.4$ \\
\hline Product C & 5 & 104.540 & 1.632 & 0.730 & $102.514-106.566$ & $102.9-106.9$ \\
\hline Product D & 5 & 99.160 & 2.393 & 1.070 & $96.188-102.132$ & $97.1-103.3$ \\
\hline Overall & 20 & 101.710 & 3.934 & 0.880 & $99.869-103.551$ & $97.0-109.4$ \\
\hline
\end{tabular}

SD: standard deviation; SE: standard error, Cl: confidence interval.

Table 4. ANOVA Results for Percent Release of Clavulanic Acid from Co-Amoxiclav Tablets (625 mg)

\begin{tabular}{|l|c|c|c|c|c|}
\hline & Sum of Squares & df & Mean Square & F & Sig. \\
\hline Between Groups & 233.554 & 3 & 77.851 & 20.615 & 0.0001 \\
\hline Within Groups & 60.424 & 16 & 3.776 & & \\
\hline Total & 293.978 & 19 & & & \\
\hline
\end{tabular}

ANOVA: one-way analysis of variance; df: degree of freedom; F: $F$ is a ratio of two mean square values.

Dissolution performance results were checked against other physical parameters like disintegration time and hardness, as these factors significantly $(p<0.05)$ affect the dissolution rate and release of drug from the finished pharmaceutical dosage formulation. Mean disintegration time was the same for both the innovator product $A$ and generic product $D$, which is consistent with dissolution characteristics for those samples. Generic products $B$ and $C$ showed abnormal disintegration times with respect to their dissolution profiles, probably because of more hydrophobic excipients present in Product B and relatively more hydrophilic excipients for Product C (Table 5).

Mean hardness data suggest that generic product $D$ possessed the least hardness, and hence had the most efficient drug release among all samples. Products $B$ and $C$ showed similar hardness properties; thus, their drug release was somewhat similar to each other, but less 
than product $A$ and $D$. The innovator product exhibited hardness that was dissimilar to all samples, although its dissolution was above the tolerance limit, which was probably due to differences in product formulation parameters (Table 6).

Table 5. Disintegration Results for Innovator (A) and Generic (B-D) of Co-Amoxiclav Tablets (625 mg)

\begin{tabular}{|c|c|c|c|c|}
\hline \multirow{2}{*}{ Sample No. } & \multicolumn{4}{|c|}{ Disintegration Time (min) } \\
\cline { 2 - 5 } & A & B & C & D \\
\hline 1 & 13 & 14 & 10 & 12 \\
\hline 2 & 12 & 14 & 10 & 12 \\
\hline 3 & 12 & 13 & 11 & 13 \\
\hline 4 & 12 & 14 & 11 & 12 \\
\hline 5 & 12 & 14 & 11 & 12 \\
\hline Mean & $12.2 \pm 0.3$ & $13.8 \pm 0.2$ & $10.6 \pm 0.3$ & $12.2 \pm 0.4$ \\
\hline
\end{tabular}

Table 6. Hardness Results for Innovator (A) and Generic (B-D) of Co-Amoxiclav Tablets (625 mg)

\begin{tabular}{|c|c|c|c|c|}
\hline \multirow{2}{*}{ Sample No. } & \multicolumn{4}{|c|}{ Hardness (kgf) } \\
\cline { 2 - 5 } & A & B & C & D \\
\hline 1 & 32.6 & 33.2 & 29.6 & 22.6 \\
\hline 2 & 29.1 & 31.5 & 27.7 & 23.8 \\
\hline 3 & 30.5 & 28.1 & 28.4 & 22.4 \\
\hline 4 & 28.7 & 26.9 & 26.9 & 24.3 \\
\hline 5 & 29.3 & 28.7 & 27.4 & 24.5 \\
\hline Mean & $30.0 \pm 0.4$ & $29.7 \pm 0.2$ & $27.9 \pm 0.2$ & $23.5 \pm 0.1$ \\
\hline
\end{tabular}

\section{CONCLUSION}

The chosen products were the most prescribed generic brands of co-amoxiclav and are used as a broad-spectrum antibiotic. The tested generic brands of this antibiotic were found to be substandard for amoxicillin release. Differences in dissolution performance of generic products may reduce the therapeutic effect of the drug.

\section{CONFLICT OF INTEREST}

The authors disclosed no conflict of interest associated with this article.

\section{REFERENCES}

1. Neu, H. C. Antimicrobial activity and human pharmacology of amoxicillin. J. Infect. Dis. 1974, 129, S123-S131. DOI: 10.1093/infdis/129.supplement_2.S123.

2. <1226> Verification of Compendial Procedures.In The United States Pharmacopeia and National Formulary USP 37-NF 32; The United States Pharmacopeial Convention, Inc.: Rockville, MD, 2014.

3. Brogden, R. N.; Carmine, A.; Heel, R. C.; Morley, P. A.; Speight, T. M.; Avery, G. S. Amoxycillin/clavulanic acid: a review of its antibacterial activity, pharmacokinetics and therapeutic use. Drugs 1981, 22, 337-362. DOI: 10.2165/00003495-198122050-00001.

4. Geddes, A. M.; Klugman, K. P.; Rolinson, G. N. Introduction: historical perspective and development of amoxicillin/clavulanate. Int. J. Antimicrob. Agents 2007, 30, S109-S112. 
DOI: 10.1016/j.ijantimicag.2007.07.015.

5. World Health Organization Expert Committee. The Selection and Use of Essential Medicines; WHO Technical Report Series, No. 994, Geneva: World Health Organization, 2015.

6. Shah, V. P. Dissolution: a quality control test vs. a bioequivalence test. Dissolut. Technol. 2001, 8, 6-7. DOI: 10.14227/DT080401P6.

7. Emami, J. In vitro-in vivo correlation: from theory to applications. J. Pharm. Pharm. Sci. 2006, 9, 169-189.

8. Dressman, J. B.; Amidon, G. L.; Reppas, C.; Shah, V. P. Dissolution testing as a prognostic tool for oral drug absorption: immediate release dosage forms. Pharm. Res. 1998, 15, $11-$ 22. DOI: $10.1023 / A: 1011984216775$.

9. Woodcock, J. The concept of pharmaceutical quality. Am. Pharm. Rev. 2004, 7, 10-15.

10. Cardot, J.; Beyssac, E.; Alric, M. In vitro-in vivo correlation: importance of dissolution in IVIVC. Dissolut. Technol. 2007, 14, 15-19. DOI: 10.14227/DT140107P15.

11. Amidon, G. L.; Lennernäs, H.; Shah, V. P.; Crison, J. R. A theoretical basis for a biopharmaceutic drug classification: the correlation of in vitro drug product dissolution and in vivo bioavailability. Pharm. Res. 1995, 12, 413-420. DOI: 10.1023/A:1016212804288.

12. Salvo, F.; De Sarro, A.; Caputi, A. P.; Polimeni, G. Amoxicillin and amoxicillin plus clavulanate: a safety review. Expert Opin. Drug Saf. 2009, 8, 111-118. DOI: $10.1517 / 14740330802527984$.

13. Olanrewaju, O.; Paul, A.; Olusola, A. Quality assessment of amoxicillin-clavulanate potassium tablets in Lagos, Nigeria. J. Chem. Pharm. Res. 2012, 4, 5032-5038.

14. Ambay, T. M.; Kahaliw, W.; Somasundaram, J. In-vitro quality evaluation of different products of amoxicillin-clavulanate potassium tablets. Int. J. Al. Med. Sci. Clin. Res. 2016, 4, 10-16. DOI: 10.13140/RG.2.1.1247.9608. 